\title{
Assessing the Potential of Catch-Only Models to Inform on the State of Global Fisheries and the UN's SDGs
}

\author{
Rishi Sharma ${ }^{1, * \mathbb{D}}$, Henning Winker ${ }^{2} \mathbb{D}$, Polina Levontin ${ }^{3} \mathbb{D}$, Laurence Kell ${ }^{3}$, Dan Ovando ${ }^{4}$, \\ Maria L. D. Palomares ${ }^{5}$, Cecilia Pinto ${ }^{2}$ and Yimin Ye ${ }^{1}$ (I) \\ $1 \quad$ FAO Viale Del Terme de Caracella, 00153 Rome, Italy; yimin.ye@fao.org \\ 2 Joint Research Centre, European Commission, 21027 Ispra, Italy; Henning.WINKER@ec.europa.eu (H.W.); \\ cecilia.pinto@ec.europa.eu (C.P.) \\ 3 Centre for Environmental Policy, Imperial College London, London SW7 2BX, UK; \\ polina.levontin02@imperial.ac.uk (P.L.); 1.kell@imperial.ac.uk (L.K.) \\ 4 SAFS, University of Washington, Seattle, WA 98195, USA; danovan@uw.edu \\ 5 Sea Around Us, University of British Columbia, Vancouver, BC V6T 1Z4, Canada; \\ m.palomares@oceans.ubc.ca \\ * Correspondence: rishi.sharma@fao.org
}

Citation: Sharma, R.; Winker, H.; Levontin, P.; Kell, L.; Ovando, D.; Palomares, M.L.D.; Pinto, C.; Ye, Y. Assessing the Potential of Catch-Only Models to Inform on the State of Global Fisheries and the UN's SDGs. Sustainability 2021, 13, 6101. https:// doi.org/10.3390/su13116101

Academic Editors: Vincenzo Torretta, Eun-Sung Chung, António

Dinis Ferreira, Peter Goethals and Jose Navarro Pedreño

Received: 8 April 2021

Accepted: 24 May 2021

Published: 28 May 2021

Publisher's Note: MDPI stays neutral with regard to jurisdictional claims in published maps and institutional affiliations.

Copyright: (c) 2021 by the authors. Licensee MDPI, Basel, Switzerland. This article is an open access article distributed under the terms and conditions of the Creative Commons Attribution (CC BY) license (https:// creativecommons.org/licenses/by/ $4.0 /)$.

\begin{abstract}
Catch-only models (COMs) have been the focus of ongoing research into data-poor stock assessment methods. Two of the most recent models that are especially promising are (i) CMSY+, the latest refined version of CMSY that has progressed from Catch-MSY, and (ii) SRA+ (Stock Reduction Analysis Plus), one of the latest developments in the field. Comparing COMs and evaluating their relative performance is essential for determining the state of regional and global fisheries that may be lacking necessary data that would be required to run traditional assessment models. In this paper we interrogate how performance of COMs can be improved by incorporating additional sources of information. We evaluate the performance of COMs on a dataset of 48 data-rich ICES (International Council for the Exploration of Seas) stock assessments. As one measure of performance, we consider the ability of the model to correctly classify stock status using FAO's 3-tier classification that is also used for reporting on sustainable development goals to the UN. Both COMs showed notable bias when run with their inbuilt default heuristics, but as the quality of prior information increased, classification rates for the terminal year improved substantially. We conclude that although further COM refinements show some potential, most promising is the ongoing research into developing biomass or fishing effort priors for COMs in order to be able to reliably track stock status for the majority of the world's fisheries currently lacking stock assessments.
\end{abstract}

Keywords: SDG 14.4.1; SOFIA; overfishing; sustainable; stock reduction; SRA+, CMSY+

\section{Introduction}

In the past few years, multiple new computational approaches to assess stock status in data-limited situations have been developed [1-11]. These new methods are invaluable to fishery managers around the world and agencies such as the Food and Agriculture Organization of the United Nations (FAO) that are tasked under the United Nation's (UN) Sustainable Development Goals (Target 14) with tracking global progress on sustainable use of fisheries resources. The FAO (2020) already performs a systematic assessment with 445 stocks on a biannual basis which covers approximately $70 \%$ of the global landing records, yet many smaller stocks are still missing from these analyses due to data deficiencies (landing records on their own are insufficient for traditional stock assessment models that require also biological and fisheries effort data, and for many of the world's stocks landing data themselves are unavailable). The fact remains that a large number of the world's stocks lack formal assessments and because their status is 'unknown' they are generally omitted from calculating indicators of overfishing such as SDG14.4.1—a UN-agreed 
country-specific indicator of overfishing which counts the proportion of stocks that are biologically sustainable, but only among the stocks with a 'known' or quantitatively assessed stock status. Therefore, gaps in both data and methodology contribute to uncertainty over the real state of many of the world's fisheries, and impede the measurement of progress towards achieving the UN's sustainable development goals. Providing quantitative assessment of stock status requires some form of data on individual fisheries, and catch histories maintained by the FAO are in many cases the only data type available for fisheries large and small across the world [8]. As such, a growing number of 'catch-only' models (COMs) have been developed in an effort to narrow the assessment gap between data-rich and data-poor fisheries. Given the increased dependence of global fishery assessments on COMs, it is imperative to assess the reliability of these tools as well as explore ways in which their performance might be improved. That is the central aim of this paper.

When data are limited, the structure of a given assessment model has relatively greater influence on assessment outcomes than the data. One consequence of this is that different methodologies applied to the same dataset can produce disconcertingly divergent conclusions, as model assumptions, rather than data, shape modeling results. This is exactly what we have seen when it comes to global assessments of the health of fish stocks, and this is what we would expect more generally in other model-based assessments, e.g., those that are used to measure SDGs, when data are insufficiently robust. Different teams of scientists relying on the same public databases of catch data using different methods (that make different assumptions and aggregate data in different ways) have variously concluded that around two-thirds of global stocks are overfished [12] or that one-third are (FAO). FAO's world assessment relies mostly on 'traditional' full statistical stock assessments, but also some data-limited assessments or expert elicitation methods [13], and it is possible that the selection bias in favor of larger stocks with formal assessments is behind their relatively optimistic, e.g., compared to Worm's, global outlook on the world's fisheries. In addition, FAO's methodology tends to aggregate stocks into larger units versus the Worm et al. approach and that can be a factor explaining the differences. There is evidence that smaller, often unassessed, stocks may be in poorer condition on average. Therefore, by aggregating data and by not being able to take these smaller unassessed stocks into account, the FAO's and hence the UN's global estimates of stock status may be positively biased $[14,15]$. Bringing more of the world's stocks into assessment would reduce these biases, and enable more informed decision making and accountability, helping to achieve the UN's sustainability goals.

Many of the stocks that are not yet assessed might be included in the global FAO assessments if the methodology of combining existing catch data and informative priors is sufficiently developed, validated and disseminated. In the current global toolbox, there are both model-based and model-free approaches that use primarily catch data to assess stock status. Model-free approaches include stock status plots, which might use catch time series to assign development stages to individual stocks based on catch levels in relation to the maximum/peak catch of the time series, e.g., [16-18]. However, these methods have been criticized for their lack of theoretical underpinnings and the failure to utilize the knowledge of life history or dynamics [19]. As a result, various catch-only model-based approaches (COMs) that incorporate formal assumptions about population dynamics were developed for data-limited stocks [15,20,21]. In contrast to model-free approaches, COMs are designed to analyze catch time series in conjunction with existing prior knowledge about the stock's resilience and exploitation history as well as general understanding about population dynamics. Among these methods are depletion-corrected average catch models [22] and depletion-based stock reduction analysis [20]. Carruthers et al. [1] tested the performance of different catch-based MPs with different life history traits and found catch-only models often performed poorly as compared to simple indicatorbased approaches in the context of fisheries management algorithms for decision making (on allowable catches or sustainable levels of fishing effort). Sun et al. [2] also looked at different data-limited management procedures (MPs) to test whether they can achieve 
sustainable fishery targets similar to data-rich scenarios and found that length-based and SPR (Spawning Potential Ratio)-based approaches could perform well with data-poor indicators. These studies provide contrasting performance evaluations of catch-based and other types of data-limited models in the context of fisheries management. While COMs are considered to be data-limited, some of the stock parameters required for these methods to function reliably would be too difficult to obtain for the majority of global stocks. This paper attempts to clarify when should COMs be used and when information might be insufficient, and alternatives, perhaps qualitative methods, might be preferred.

\section{Materials and Methods}

The two models CMSY+ and SRA+ evolved from the early COMs Catch-MSY [21] and CMSY [23]. The core of SRA+ is a generalized Pella-Tomlinson [24] production model, which is purposefully simplified to Schaefer model in the case of CMSY+ [23]. While such simplified models abstract away many important details of fish biology and fleet behavior, they are the highest-resolution models that the data-limited cases will support. By contrast, traditional stock assessment methods are able to parameterize (for data-rich stocks) and model sophisticated biological and socioeconomic processes. The purpose of COMs is not to make substantial improvements in the fitting of surplus production models, but to provide flexible tools for improving estimates with additional sources of information. Growth rates can become unrealistically large when the population reaches low sizes under the Pella-Tomlinson model. CMSY+ and SRA+ deals with this problem by following the methods described in $[23,25]$ to reduce the production rate of the population when it falls below a threshold of $25 \%$ of carrying capacity.

We allow for process error in the manner of the stochastic stock reduction analysis error suggested by [26]. Process errors enable the population dynamics to deviate from the exact values given by the Pella-Tomlinson model, while still conforming to the assumptions of this model on average. Incorporation of process errors is useful for two reasons: (1) when the model is trying to fit an abundance index, process errors can reduce bias arising from lack of fit in a deterministic SRA whenever dynamics are poorly explained by catch history alone, and (2) with or without an abundance index (or other auxiliary information), the stochastic portion is necessary to get plausible uncertainty intervals in the final estimates [27].

All estimates are Bayesian in nature and fall into two distinct categories: estimated with data and without. By 'data', we refer to measurements which are used to confront model estimates within a likelihood function. These might include (in addition to catch data) fishery-independent survey data, or a CPUE index. When there is no additional data, the model resorts to filtering priors through the model equations, along with any fixed parameters and catch data. Under this mode, the model is essentially a stock-reduction analysis model [21,26], which searches for combinations of prior probability distributions that do not crash the population, given the constraints of the population dynamics model and the catches. This process updates the prior distribution of population parameters by eliminating combinations of priors that are impossible for a given catch history and a specified functional form of the production model. To ensure that the algorithms were the only difference in the 2 COMs, all inputs were the same for both CMSY+ and SRA+.

\subsection{Data}

To find suitable data and knowledge-rich stocks, we sorted through the International Council for Exploration of the Seas (ICES) SAG (stock assessment graphs) database published at the beginning of 2020 that contained ICES stock assessments for 2019. Among these we selected 48 stocks that had data-rich age-structured assessments based on traditional analytical model frameworks (e.g., SAM, XSA, and Stock Synthesis [28]). As biomass that provides maximum sustainable yield ( $\left.\mathrm{B}_{\mathrm{MSY}}\right)$ is not always estimated by ICES, a proxy to $\mathrm{B}_{\mathrm{MSY}}$ was consistently used, equal to $3^{*}$ Blim, where Blim is a limit reference point based on the value of spawning stock biomass (SSB) below which the stock is considered to have 
reduced reproductive capacity. It is derived in post-processing from the breakpoint of a segmented regression fitted to the SSB-recruitment pairs estimated by the ICES stock assessment. Figure 1 shows the stock trajectories relative to $B_{\text {MSY. }}$. It can be seen that stocks tend to share a common trend, mainly due to the adoption of the maximum sustainable yield (MSY) approach by ICES after 2008.

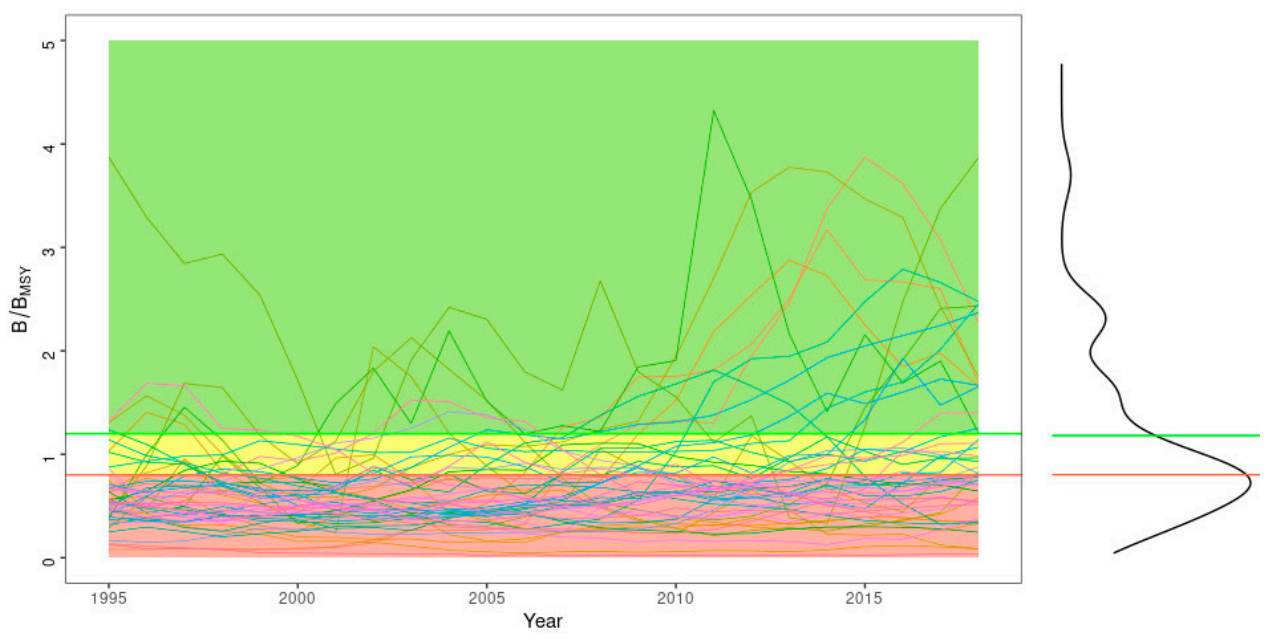

Figure 1. Trajectories of biomass of 48 International Council for Exploration of the Seas (ICES) stocks chosen for this analysis relative to biomass that provides maximum sustainable yield (B $\mathrm{B}_{\mathrm{MSY}}$ ), estimated from traditional stock assessments. The red horizontal line indicates $80 \%$ of $B_{\text {MSY }}$ below which the Food and Agriculture Organization of the United Nations (FAO) considers stocks to be overfished; the green horizontal line $\left(120 \%\right.$ of $\left.\mathrm{B}_{\mathrm{MSY}}\right)$ indicates where stocks are underfished. The density on the right represents final year estimates.

According to the FAO classification, the majority of these 48 stocks were overfished (B/ $\mathrm{B}_{\mathrm{MSY}}$ is less than 0.8) for most of the time series, (Figure 1). Among those 48 stocks, there were 3 families of fish: Clupeidae (herring, sardines and sprat), Gadidae (cod, haddock, hake and saithe), and Pleuronectidae (plaice and halibut). This highlights an issue that these are the families for which informative priors for life history parameters are readily available (e.g., through databases such as FishBase) while for other, less studied families of species/invertebrates this data may not exist. Note that in our scenarios the levels of uncertainty in the intrinsic rate of population increase (r) varied between CV of $10-30 \%$ depending on the knowledge of the life history parameters ( $\mathrm{r}$ estimates were taken from FishBase database and adjusted in COMs based on the production function).

As most global fisheries that lack traditional assessments do not have a long time series of data (as can be glimpsed from RAM Legacy Stock Assessment Database or FAO Global Fisheries Database), to approximate data-poor fisheries we limited the analysis to the last 25 years of landings. Among the stocks selected, there is a wide variation in terms of levels of catch and some species exhibit large swings in relative abundance, temporarily overshooting $\mathrm{B}_{\mathrm{MSY}}$ by up to fourfold (Figure 1). For the purposes of evaluating COMs, we used the existing traditional assessment results that incorporate all available information into ICES data as the 'true' state of the stocks, acknowledging that the values produced by the ICES assessments are themselves model estimates containing their own uncertainty.

\subsection{Scenarios}

For each of the two COMs methods, SRA+ and CMSY+, we ran six scenarios that represented different kinds of informational inputs, where the default 'naive' settings represented a high level of uncertainty corresponding to large confidence bounds. The six scenarios are described in Table 1. These scenarios were chosen from combinations of three sources of inputs: an index of abundance, a prior for the initial level of depletion and a 
prior for the depletion in the final year. Scenarios were identified by six distinct names ("Scenario Name" column in Table 1): naive, naive2, cr2, exp, bsm1 and bsm2.

Table 1. Six scenarios corresponding to different informational inputs into the two COMs, the SRA+ and $\mathrm{CMY}+$.

\begin{tabular}{|c|c|c|c|c|}
\hline Description & Scenario Name & $\begin{array}{l}\text { Initial Depletion } \\
\text { Prior (Initial B/K) }\end{array}$ & $\begin{array}{l}\text { Final Year } \\
\text { Depletion Prior } \\
\text { (End } \mathrm{B} / \mathrm{K})\end{array}$ & $\begin{array}{c}\text { Auxiliary Indices } \\
\text { of Abundance } \\
\text { (Index) }\end{array}$ \\
\hline Default Settings & naive $(\mathrm{HH})$ & Heuristic & Heuristic & None used \\
\hline $\begin{array}{c}\text { Added Knowledge } \\
\text { on Initial } \\
\text { Depletion }\end{array}$ & naive2 $(\mathrm{EH})$ & Expert & Heuristic & None used \\
\hline $\begin{array}{c}\text { Added Knowledge } \\
\text { on Initial } \\
\text { Depletion and } \\
\text { Modified Final } \\
\text { Depletion Rule }\end{array}$ & cr2 (EC) & Expert & Catch Rule & None used \\
\hline $\begin{array}{c}\text { Good Knowledge } \\
\text { on Initial and Final } \\
\text { Depletion }\end{array}$ & $\exp (E E)$ & Expert & Expert & None used \\
\hline $\begin{array}{c}\text { Added } \\
\text { Informative Index } \\
\text { of Abundance }\end{array}$ & bsm1 (HI) & Heuristic & None used & Yes \\
\hline $\begin{array}{c}\text { Added } \\
\text { Informative Index } \\
\text { of Abundance and } \\
\text { Knowledge of } \\
\text { Initial Depletion }\end{array}$ & bsm2 (EI) & Expert & None used & Yes \\
\hline
\end{tabular}

Four scenarios did not rely on any index of abundance and two scenarios (bsm1 and bsm2) used an index of abundance based on perfect knowledge (congruent with ICES assessments that represent 'the truth' in our evaluations). This represents an extremely idealized scenario where not only an index of abundance is available but it is unbiased and precise. Two different types of priors were considered for initial depletion level (Initial $\mathrm{B} / \mathrm{K})$, one representing a prior obtained through expert elicitations (EH, EC, EE, EI) and one based on a formal heuristic method (HH, HI). Again, in the case of expert priors, it was assumed that 'the expert' has good albeit somewhat uncertain knowledge. The expert priors were constructed as follows, in order to mimic good expert knowledge: if the stock is severely depleted (according to the ICES assessment), then 'the expert' is assumed to guess that the average biomass is 0.18 of unfished equilibrium $\left(\mathrm{B}_{0}\right)$, with uncertainty in the expert prior always modeled by using a CV of at least $20 \%$; if the 'true' state corresponds to intermediate depletion, then the guess is 0.38 ; if indeed it is fished at optimal levels, expert prior average is 0.58 ; and if the stock is underfished, the expert prior average is set to 0.87 of unfished biomass.

Heuristic priors and catch-rule priors were constructed based on various algorithms using available catch data and other external sources such as life history parameters from publicly available databases such as FishBase. One of the methods for an initial heuristic depletion prior is similar to the one identified by Rosenburg et. al. [8]). For the final depletion prior, we used the following:

\subsection{Heuristic Priors}

If the final catch is greater than $50 \%$ of the maximum catch, then the final depletion is between $30 \%$ and $70 \%, 1-50 \%$ otherwise.

\subsection{Catch-Rule Priors}

If the final year catch is between $30 \%$ and $70 \%$ of the maximum catch, a prior centered around $40 \%$ of the catch is used instead of default mean; if the final year catch is greater 
than $70 \%$ of the peak, then the default prior's mean is set to $70 \%$; and if the final year catch is lower than $30 \%$ of the maximum, then the default prior is centered at $20 \%$. All these priors have a CV of 0.2 and are based on the initial CMSY paper $[8,21]$.

\section{Results}

First, we looked at how well these methods were able to capture the big picture, and describe the historic evolution of the fishery at an aggregate level (see Figure 2 for SRA+ and Figure 3 for $\mathrm{CMSY}+$ ).

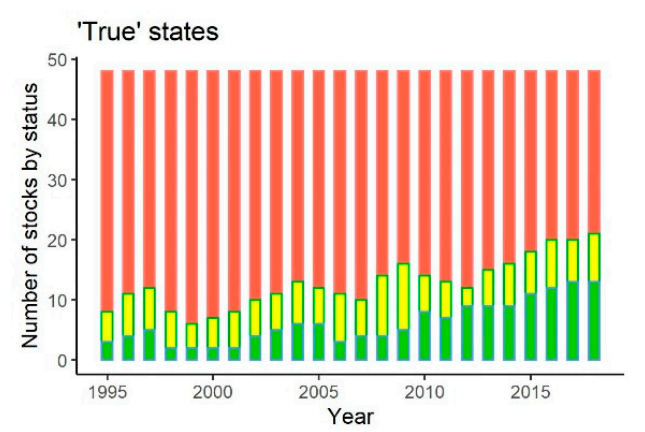

SRA+ 'naive' scenario estimates defaults

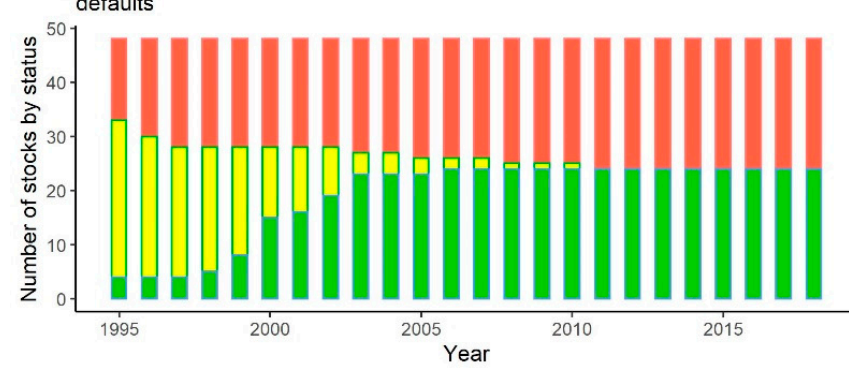

SRA+ 'cr2' scenario estimates

catch-rule for final depletion plus good knowledge of initial depletion

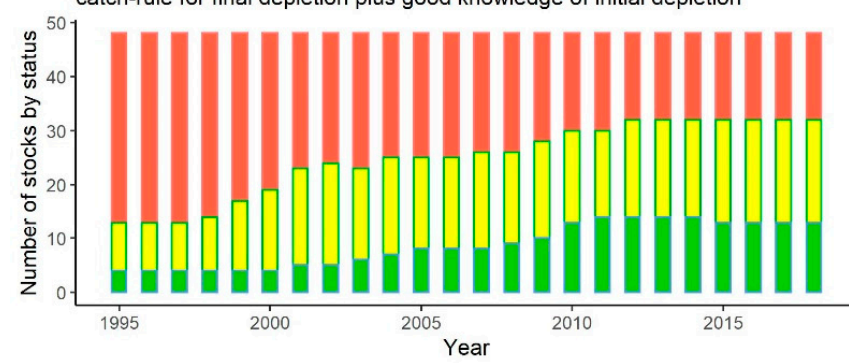

SRA+ 'bsm1' scenario estimates

a reliable index of abundance

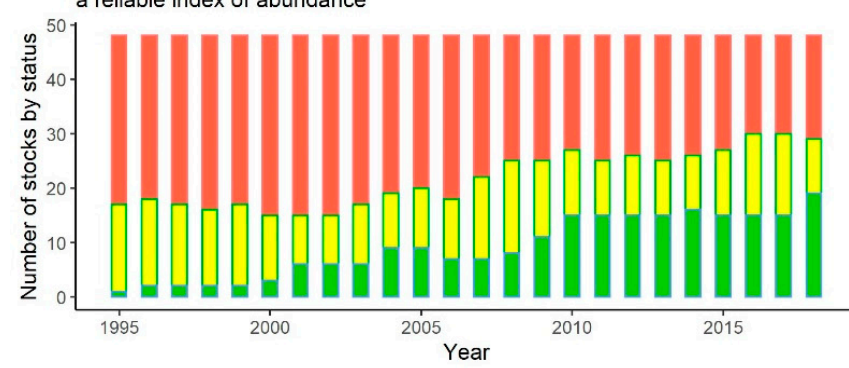

SDG14.4.1 'true' proportion of stocks that are not overfished (e.g. not SRA+ estimates of SDG14.4.1 in the final year are shown in spaced coloured text

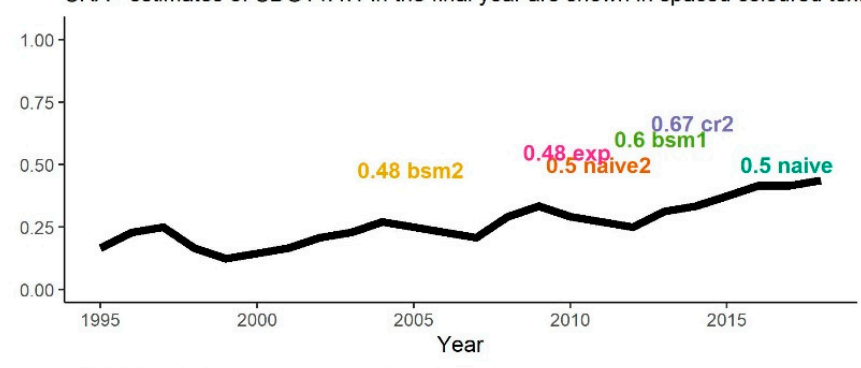

SRA+ 'naive2' scenario estimates added good knowledge of initial depletion

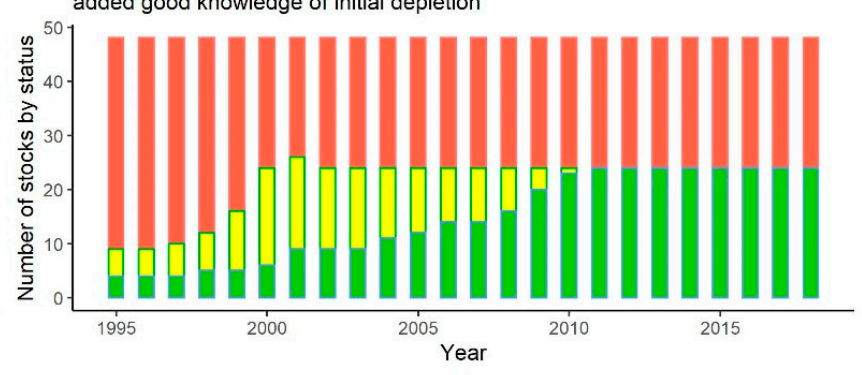

SRA+ 'exp' scenario estimates

good knowledge of both initial and final depletion

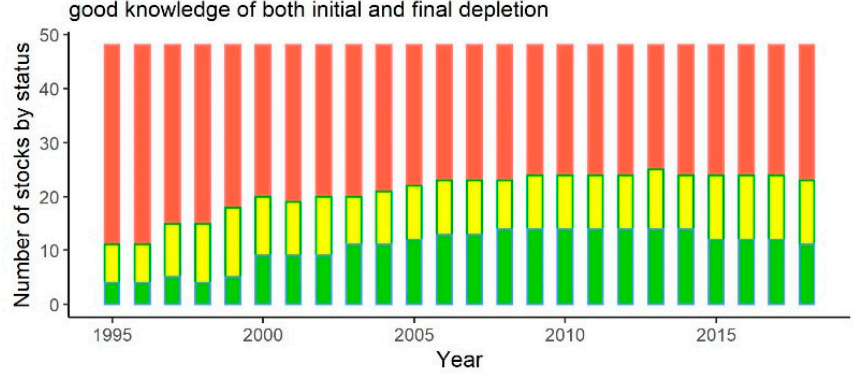

SRA+ 'bsm2' scenario estimates

a reliable index of abundance plus good knowledge of initial depletion

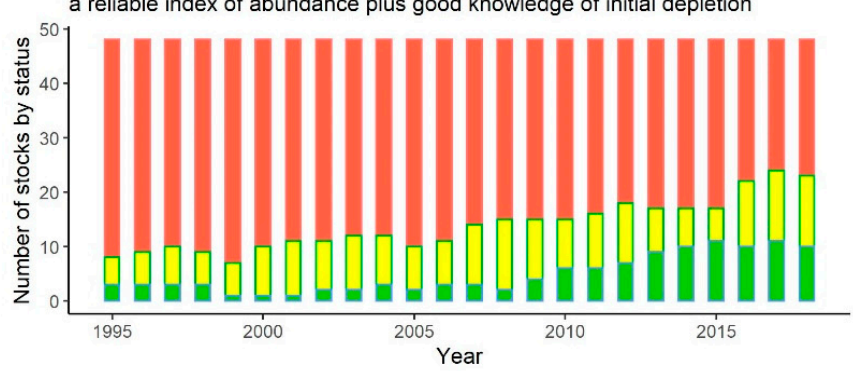

Figure 2. Comparing the 'true' evolution of stocks over time (top left bar chart) with six different attempts by SRA+ model to estimate it, under different prior knowledge scenarios as described in Table 1. The bars show proportions of stocks that are underfished (above 1.2 $\mathrm{B}_{\mathrm{MSY}}$, in green), sustainably fished (assessed to be between 0.8 and 1.2 of $\mathrm{B}_{\mathrm{MSY}}$, in yellow) and overfished ( $<0.8 \mathrm{~B}_{\mathrm{MSY}}$, in red) for each year. The final year SRA+ estimates are marked by colored text (horizontally spaced for legibility) while the 'true' proportion of sustainably fished stocks is shown by the thick black line (top right graph). 

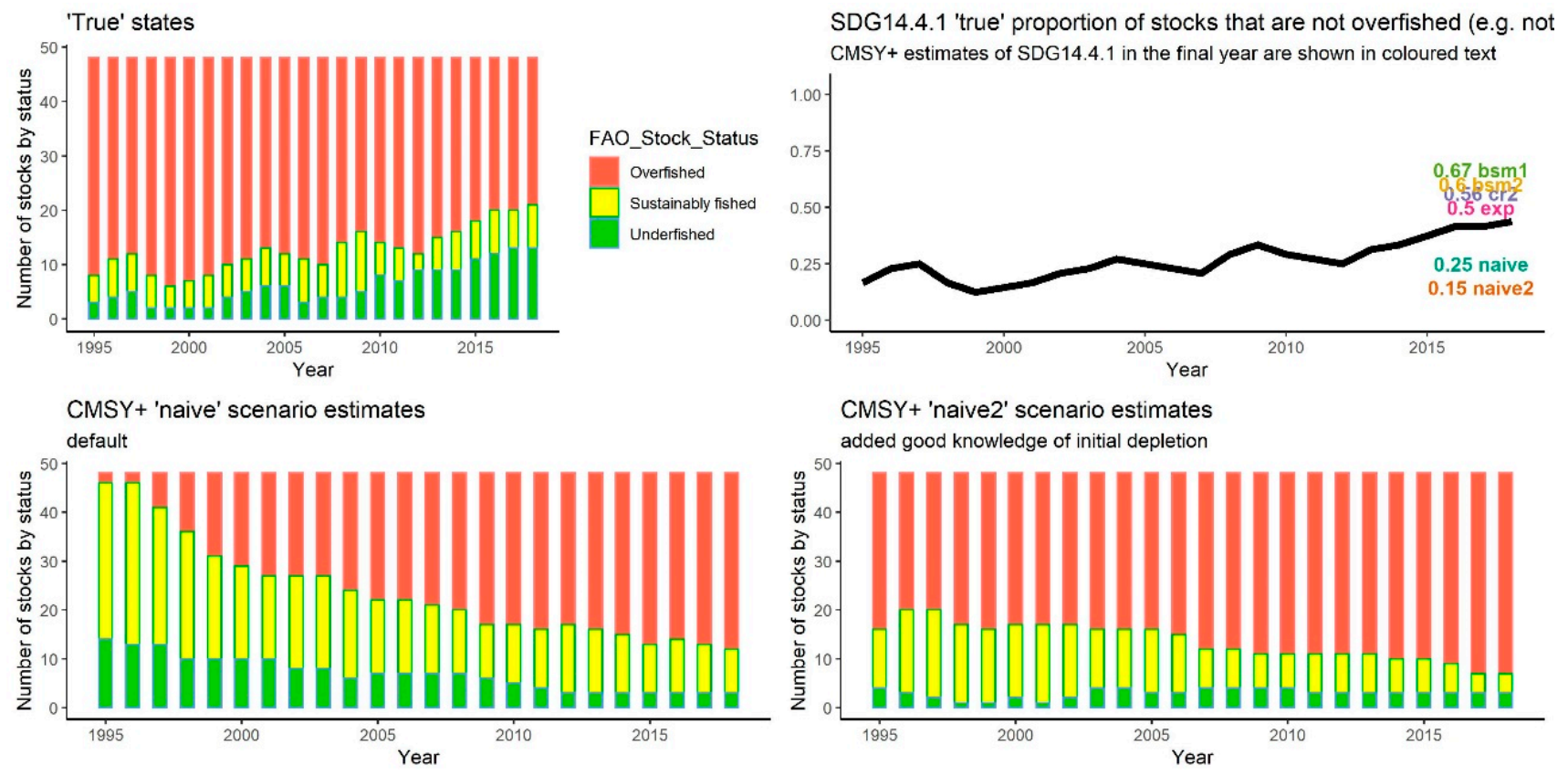

CMSY+ 'cr2' scenario estimates

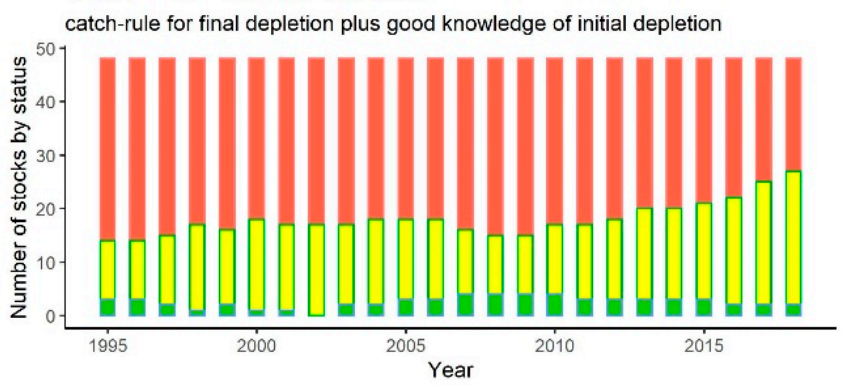

CMSY+ 'exp' scenario estimates

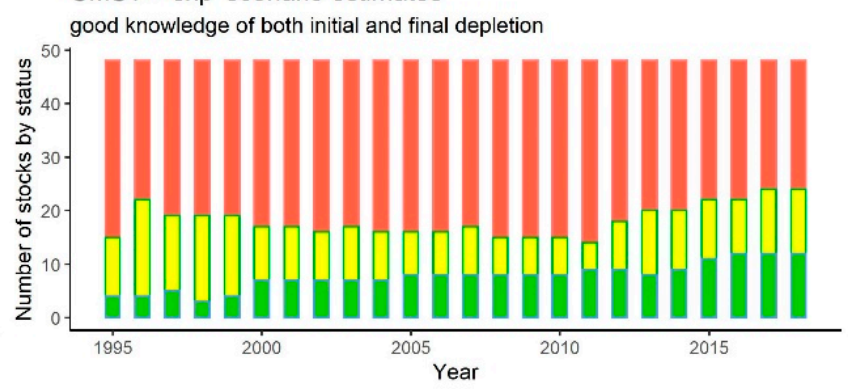

CMSY+ 'bsm1' scenario estimates

a reliable index of abundance

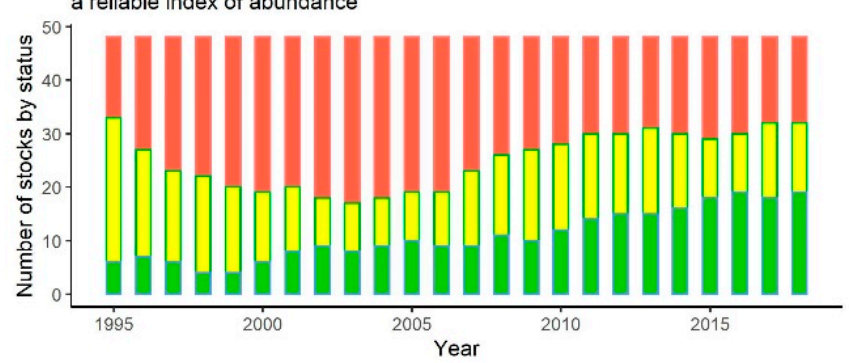

CMSY+ 'bsm2' scenario estimates

a reliable index of abundance plus good knowledge of initial depletion

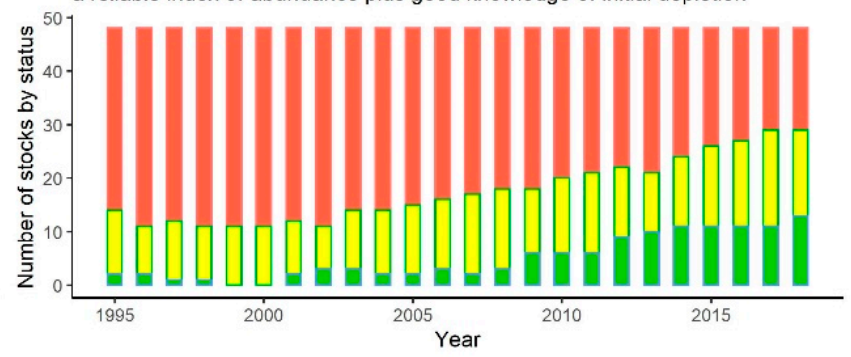

Figure 3. Comparing the 'true' evolution of stocks over time (top left bar chart) with six different attempts by CMSY+ model to estimate it, under different prior knowledge scenarios as described in Table 1. The bars show proportions of stocks that are underfished (above $1.2 \mathrm{~B}_{\mathrm{MSY}}$, in green), sustainably fished (assessed to be between 0.8 and 1.2 of $\mathrm{B}_{\mathrm{MSY}}$, in yellow) and overfished ( $<0.8 \mathrm{~B}_{\mathrm{MSY}}$, in red) for each year. The final year CMSY+ estimates are marked by colored text (horizontally spaced for legibility) while the 'true' proportion of sustainably fished stocks is shown by the thick black line (top right graph). 
At a first glance, it does not seem that SRA+ can capture the dynamics particularly well. Only in the 'bsm2' and 'exp' scenarios, which correspond to having a very good guess about initial depletion and either a superb index of abundance (good expert knowledge of biomass dynamics over time) in the case of 'bsm2' or final depletion in the case of 'exp', can it be considered to do an adequate job, although that is a subjective judgement. In all scenarios, SRA+ overestimates the final value of SDG 14.4.1: the 'exp' and 'bsm2' estimates $(\sim 48 \%)$ come closest (Figure 3$)$, while the 'cr2' scenario is the most positively biased, predicting that the proportion of sustainably fished stocks in the final year is $67 \%$ while the true value is $44 \%$.

Perhaps of less significance is that the models were only able to assess the initial depletion with a reasonable precision when given a very informative prior for that quantity. Without extra helpful knowledge of initial depletion, and especially with default heuristic as priors ('naive' scenario), the SRA+ model's assessment of the initial proportion of overfished stocks was poor. The same conclusion holds for the CMSY+ model (Figure 2); see 'naive' and 'bsm1' scenarios which lack informative priors on initial depletion.

Similarly, CMSY+ did not seem to document the evolution of the fishery to a satisfying degree, except in the case ('bsm2') when the model was given a very good hint of what was happening over time (a perfect index of abundance) and near-perfect information about what was going on at the beginning (a very informative initial depletion prior). Even in those fortunate circumstances, this method seems to be performing worse than the SRA+ model (given the same information) and, worryingly, it appears to arrive at overly optimistic conclusions. The 'true' SDG14.4.1 indicator tells us that $44 \%$ of stocks in the final year are not overfished, while SRA+ 'bsm2' estimates the percentage to be $48 \%$, and CMSY+ 'bsm2' thinks that SDG14.4.1 is $60 \%$. In terms of estimating the final proportion of stocks that are not overfished, the 'exp' scenario comes closest. For the CMSY+ its estimate of SDG14.4.1 in the final year is 50\%, only somewhat overoptimistic compared to the true estimate of $44 \%$.

Relative errors across trajectories indicate that it is possible for the methods to capture the trend in the aggregate even if individual stock trajectories are assessed with bias for most of the time series, since the bias is in both directions an average error across all stocks and might be small as biases cancel each other out (Table 2).

Table 2. Aggregate bias in B/B $\mathrm{B}_{\mathrm{MSY}}$ in the final year across all stocks for both models.

\begin{tabular}{ccccccc}
\hline Software & Naive & Naive2 & Bsm1 & Exp & Cr2 & Bsm2 \\
\hline SRA+ Aggregate Bias & $18.0 \%$ & $18.8 \%$ & $16.7 \%$ & $0.0 \%$ & $-4.2 \%$ & $2.1 \%$ \\
CMSY+ Aggregate Bias & $-25.0 \%$ & $-22.9 \%$ & $25.0 \%$ & $-4.2 \%$ & $-14.6 \%$ & $14.6 \%$ \\
\hline
\end{tabular}

This emphasizes that while these methods appear to be good for classification on the aggregations of stocks, they probably should not be used for prescriptive assessment advice on a single species in the short term. In Figure 4, we can compare how well these two best-performing scenarios, SRA+ 'bsm2' and CMSY 'bsm2', are able to estimate dynamics of individual stocks. It is clear from Figure 4 that the methods are sometimes finding it difficult to track an individual species' relative abundance over time even when given a very informative index of abundance and a spot-on guess at initial depletion ('bsm2'). For many stocks, the 'bsm2' version allows both models to track relative abundance fairly consistently. 

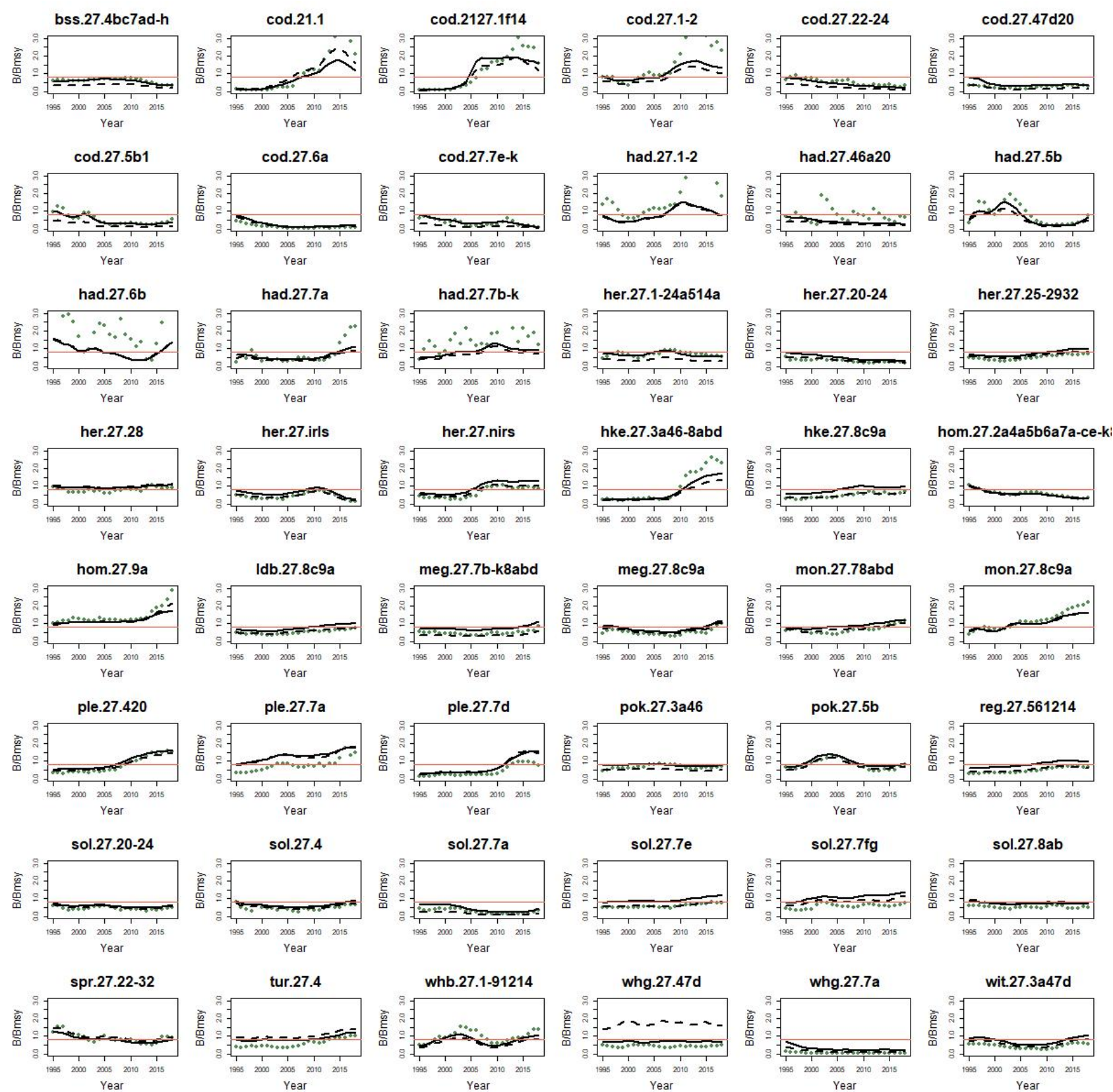

Figure 4. 'True' dynamics of B/B $\mathrm{B}_{\mathrm{MSY}}$ over time (green points) for all 48 stocks (titles: ICES stock code names) and the estimates for both SRA+ 'bsm2' (dashed line) and CMSY+ 'bsm2' (solid line) models. The horizontal red line is the FAO threshold for designating stocks as 'overfished'.

In Figures 5 and 6 we consider the sensitivity and specificity of the two models under the same six scenarios. The sensitivity of a test is the probability of correctly identifying an unwelcome condition, here overfishing, and specificity is an ability to diagnose an absence of an ill condition correctly, so correctly identifying when there is no overfishing. Type II error, or the probability of labeling an overfished stock as 'sustainable' in FAO terminology, is $100 \%$ minus sensitivity, which is shown in Figure 5. It is debatable what is the minimum sensitivity or specificity values that we would consider acceptable, but certainly the higher the better. We probably want at least a $75 \%$ chance of telling when a fishery is overfished and a similar probability for identifying correctly sustainable fisheries. This is equivalent to considering that an acceptable type II error should be less than $25 \%(100-75 \%)$, or, in other words, that fewer than 1 in 4 overfished stocks should be mislabeled as 'sustainable'. By these criteria, only SRA+ (in the 'bsm2' and 'exp' scenarios) is acceptable in terms of 
both sensitivity and specificity as it is above $75 \%$ in the final year in two of the scenarios (Figures 5 and 6). For the CMSY+ model, the scenarios that do okay on sensitivity ('naive' and 'naive2') do not perform well on specificity.
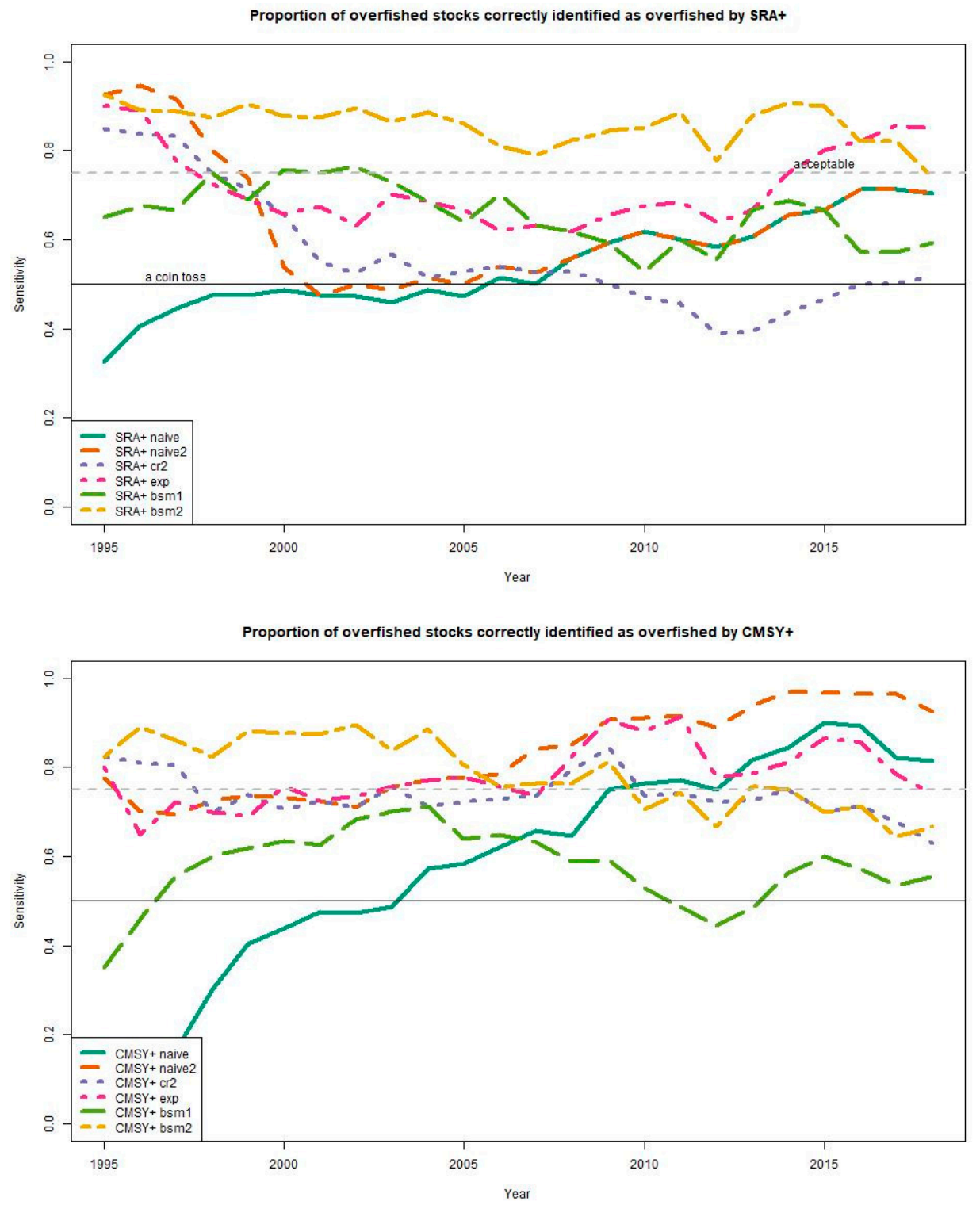

Figure 5. Proportion of overfished stocks that were correctly identified as overfished by various methods.

Of all of the scenarios explored here, only one potential method seems promising: SRA+ when it can be supplied with a good estimate of initial abundance and also provided with either a reliable index of abundance ('bsm2') or final depletion estimate ('exp'). If the $75 \%$ threshold criterion is slightly relaxed then the 'exp' scenario in CMSY+ is also doing reasonably well (it dips slightly below the threshold in the final year but does well overall). However, it is not a scientist's role to suggest acceptability criteria: the acceptability thresholds mentioned here are open to discussion and revisions; it is up to managers and stakeholders to make the final call. This leads us to cautious conclusions. 

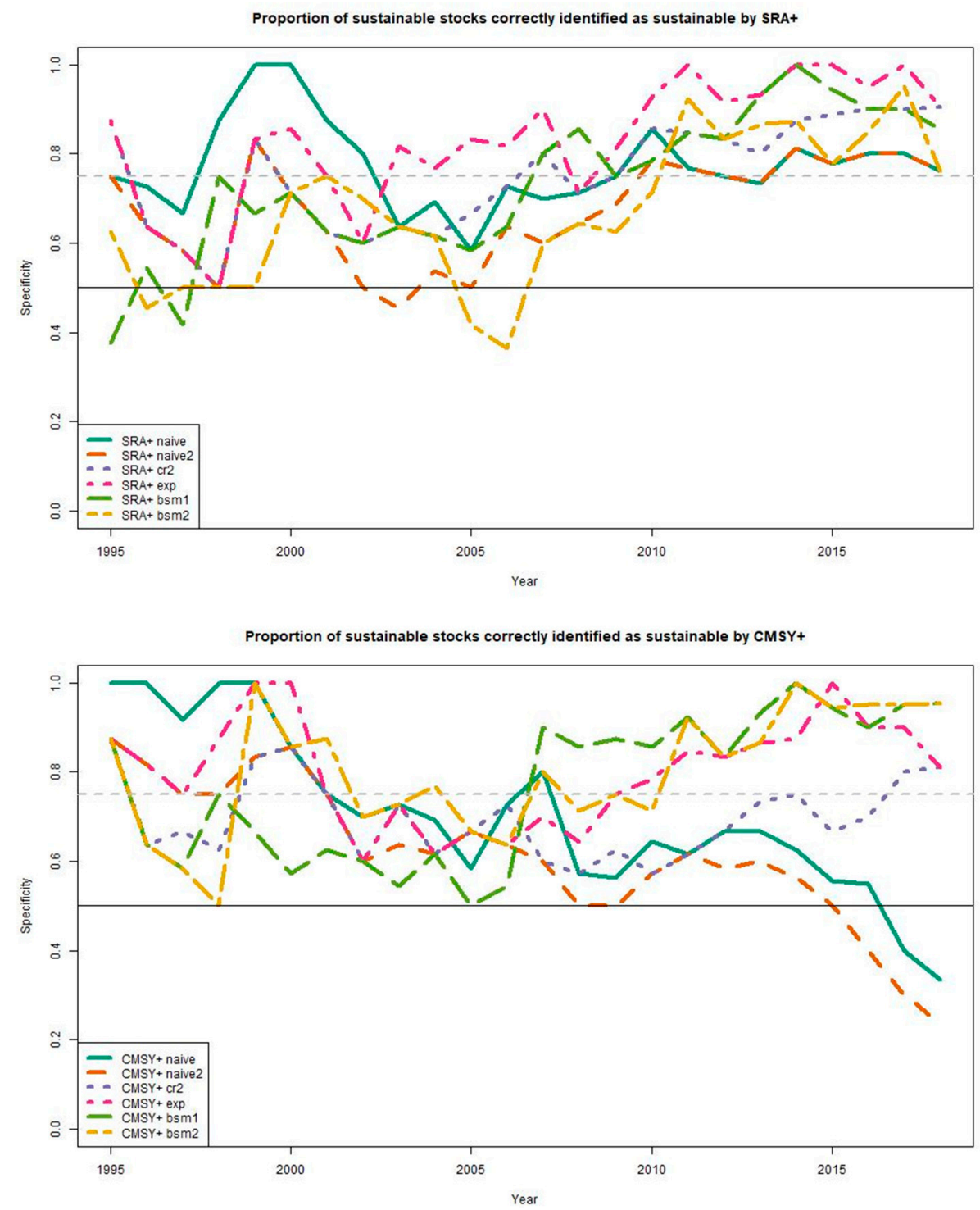

Figure 6. Proportion of not-overfished stocks that were correctly identified as not-overfished by various methods.

\section{Discussion}

While data-limited models (DLM) such as those seen in [1,2] focus on management procedures (MPs) that would ensure sustainable fisheries with simple data-poor control rules, the methods focused on here are estimating stock status in a synoptic sense.

We evaluated the ability of two COMs, SRA+ and CMSY+, to inform on the status of fisheries stocks, using varying levels of informative data. Our results corroborate those of [8], pointing to problems with much of the heuristics-based methods used in COMs, and indicate that one should not run these models using the default settings. Knowledge of the shape of the production function and informative priors are essential for getting a good estimate of depletion in the final year when using COMs. Both implementations unsurprisingly performed well if one had a good prior knowledge of the fishery for a stock. In particular, good initial depletion estimates were needed. If the time series are short, which is the case for most assessment models (e.g., Stock Synthesis in [29] or JABBA [25]), initial depletion is difficult to estimate. Approaches currently under development [29-31] indicate that using external sources of information, such as swept area ratio (SAR [29]) or a fishery management index (FMI, [32]) could provide information on current depletion. This would be especially valuable in cases where there is limited information on the history 
of the fishery. Catch data by themselves can be uninformative about changes in depletion; for example, catch could be relatively constant in a fishery where entry was unrestricted and the stock declining.

While these methods may be suitable for categorizing data-poor stocks in aggregate considering that the overall bias is relatively low when averaged for a number of stocks (Table 2), there is no substitute for field programs and direct data relevant to assessments (survey data or length data). The better the information, the better we can truly understand the dynamics of these fisheries, and act to reduce the risk to both marine environments and society. In many parts of the world, however, this may not be possible without international assistance as such fisheries data collection programs are expensive to run, and government budgets are limited and stressed by other priorities [32]. However, the long-term cost of depleting a resource may be much greater than the investment required to obtain better data and manage fisheries sustainably.

As shown in the analysis, these approaches have some potential in regional overviews even if the individual assignments are inaccurate. When combined with informative priors, COMs can provide broad advice for a region and globally. The algorithms used for classification need to be clearly documented, so one can replicate the methodologyreproducibility has been neglected in the global scale studies [10]. In addition, the potential of developing priors from external sources make these approaches attractive as their performance can be improved as soon as more data is collected on the ground.

Finally, SDG 14.4.1, a UN sustainable development goal, states: “By 2020, effectively regulate harvesting and end overfishing, illegal, unreported and unregulated fishing and destructive fishing practices and implement science-based management plans, in order to restore fish stocks in the shortest time feasible, at least to levels that can produce maximum sustainable yield as determined by their biological characteristics". COM models could be developed to report on SDG 14.4.1 on a country or regional level, providing a standardized method so we can track progress across the world in a universal manner. However, there are clearly problems when using COM models without the support of informative priors. The results of this paper support the concerns that were raised about catch-only methods in some of the previous evaluations [8,33]. In particular, it appears especially clear that the default settings for these models, even when informed by life history metaanalyses such as FishBase, should not be expected to provide reliable estimates of individual species dynamics. Under the default settings, these methods can provide a distorted picture of evolution of stocks over time on a regional level (FAO global assessments), and should not be relied upon in their default settings to provide meaningful estimates of the SDG14.4.1 indicator that the UN needs to track sustainable development goals related to overfishing. The SRA+ model seems to be positively biased, and is likely to say that a higher proportion of fish stocks are harvested sustainably than is really the case. Only when provided with informative priors does SRA+ mislabel fewer than 1 in 4 overfished stocks as 'sustainable'. However, while the SRA+ model is likely to present a more optimistic outlook, the CMSY+ model's estimates of SDG14.4.1 can be more pessimistic than reality (especially if close to default settings, when it mislabeled around 3 out of 4 sustainably fished stocks as 'overfished').

Performance of both models improves when extra knowledge is being supplied in terms of informative priors. Unsurprisingly, the most valuable thing in terms of improving overall model performance is informative priors of initial depletion combined with an excellent index of abundance. A natural recommendation follows that if alternative models are not applicable due to the lack of data, then investing in eliciting knowledge on the initial state of the stock and research that can be used to construct a plausible index of abundance would be worthwhile. Further, improving the knowledge basis on life history parameters for a wider range of fish stocks is paramount, if COMs are to find wider application, as this information is also used by the models. From this investigation, although it has various limitations, further broad conclusions can be drawn that are probably fairly robust. While, the SRA+ is marginally superior to the CMSY+ method if both sensitivity and specificity 
of the method are important, we recommend that both these methods continue to be developed and tested and alternative model options are explored. This study also raises concerns around using COMs when good information on initial depletion and index of abundance is not available, especially in the context of managing individual stocks. We found that COMs misidentify overfished stocks as 'sustainable' at a high rate. Therefore, our study lends weight to robust management practices approaches that are likely to protect marine ecosystems and the wellbeing of people dependent on them even in the absence of precise and timely information on the status of individual stocks.

Author Contributions: Conceptualization, R.S. and P.L.; Data curation, C.P.; Formal analysis, R.S., H.W., P.L., L.K. and D.O.; Funding acquisition, R.S.; Investigation, D.O., M.L.D.P. and Y.Y.; Methodology, R.S., H.W. and L.K.; Resources, R.S.; Software, R.S., D.O., H.W.; Supervision, R.S. All authors have read and agreed to the published version of the manuscript.

Funding: This research received no external funding.

Institutional Review Board Statement: Not applicable.

Informed Consent Statement: Not applicable.

Data Availability Statement: Data used in this study is publicly available from ICES.

Acknowledgments: We would like to acknowledge Ray Hilborn, Manuel Barange and Rainer Froese for inputs into this manuscript.

Conflicts of Interest: The authors declare no conflict of interest.

\section{References}

1. Carruthers, T.R.; Kell, L.T.; Butterworth, D.D.S.; Maunder, M.N.; Geromont, H.F.; Walters, C.; McAllister, M.K.; Hillary, R.; Levontin, P.; Kitakado, T.; et al. Performance review of simple management procedures. ICES J. Mar. Sci. 2015, 73, 464-482. [CrossRef]

2. Sun, M.; Li, Y.; Zhang, C.; Xu, B.; Ren, Y.; Chen, Y. Management of Data-Limited Fisheries: Identifying Informative Data to Achieve Sustainable Fisheries. N. Am. J. Fish. Manag. 2020, 40, 733-751. [CrossRef]

3. Demirel, N.; Zengin, M.; Ulman, A. First large-scale Eastern Mediterranean and Black Sea stock assessment reveals a dramatic decline. Front. Mar. Sci. 2020, 7, 103. [CrossRef]

4. Pons, M. Stock Status and Management in Tuna Fisheries: From Data-Rich to Data-Poor. Fisheries 2018. Available online: https:/ / digital.lib.washington.edu/researchworks/handle/1773/43059 (accessed on 27 May 2021).

5. Cortés, E.; Brooks, E.N. Stock status and reference points for sharks using data-limited methods and life history. Fish Fish. 2018, 19, 1110-1129. [CrossRef]

6. Anderson, S.C.; Cooper, A.B.; Jensen, O.P.; Minto, C.; Thorson, J.T.; Walsh, J.C.; Afflerbach, J.; Dickey-Collas, M.; Kleisner, K.M.; Longo, C.; et al. Improving estimates of population status and trend with superensemble models. Fish Fish. 2017, 18, 732-741. [CrossRef]

7. Christopher, M.F.; Olaf, P.J.; John, W.; Johnathan, J.D. The refined ORCS approach: A catch-based method for estimating stock status and catch limits for data-poor fish stocks. Fish. Res. 2017, 193, 60-70.

8. Rosenberg, A.A.; Fogarty, M.J.; Cooper, A.B.; Dickey-Collas, M.; Fulton, E.A.; Gutiérrez, N.L.; Hyde, K.J.W.; Kleisner, K.M.; Kristiansen, T.; Longo, C.; et al. Developing New Approaches to Global Stock Status Assessment and Fishery Production Potential of the Seas; FAO Fisheries and Aquaculture Circular No. 1086; FAO: Rome, Italy, 2014; p. 175.

9. Cope, J.M.; Punt, A. Length-Based Reference Points for Data-Limited Situations: Applications and Restrictions. Mar. Coast. Fish. 2009, 1, 169-186. [CrossRef]

10. Froese, R.; Demirel, N.; Coro, G.; Winker, H. A Simple User Guide for CMSY+ and BSM (CMSY_2019_9f.R); GEOMAR: Kiel, Germany, 2019; p. 16. Available online: http:/ / oceanrep.geomar.de/33076/ (accessed on 20 July 2020).

11. Free, C.M.; Jensen, O.P.; Anderson, S.C.; Gutierrez, N.L.; Kleisner, K.M.; Longo, C.; Minto, C.; Osio, G.C.; Walsh, J.C. Blood from a stone: Performance of catch-only methods in estimating stock biomass status. Fish. Res. 2020, 223, 105452. [CrossRef]

12. Worm, B.; Hilborn, R.; Baum, J.K.; Branch, T.A.; Collie, J.S.; Costello, C.; Fogarty, M.J.; Fulton, E.A.; Hutchings, J.A.; Jennings, S.; et al. 'Rebuiklding' global fisheries. Science 2009, 325, 578-585. [CrossRef]

13. FAO. Review of the State of World Marine Fisheries Resources; FAO Fisheries and Aquaculture Technical Paper No. 569; FAO: Rome, Italy, 2011; p. 334.

14. Costello, C.; Ovando, D.; Hilborn, R.; Gaines, S.D.; Deschenes, O.; Lester, S.E. Status and Solutions for the World's Unassessed Fisheries. Science 2012, 338, 517-520. [CrossRef]

15. Froese, R.; Zeller, D.; Kleisner, K.; Pauly, D. What catch data can tell us about the status of global fisheries. Mar. Biol. 2012, 159, 1283-1292. [CrossRef] 
16. Froese, R.; Kesner-Reyes, K. Impact of Fishing on the Abundance of Marine Species. ICES CM 12/L:12; Copenhagen, Denmark. 2002. Available online: http:/ / www.ices.dk \T1 \textgreater\{\}sites $\backslash T 1 \backslash$ textgreater\{\}pub (accessed on 27 May 2021).

17. Pauly, D. The Sea around UsProject: Documenting and Communicating Global Fisheries Impacts on Marine Ecosystems. Ambio 2007, 36, 290-295. [CrossRef]

18. Kleisner, K.; Zeller, D.; Froese, R.; Pauly, D. Using global catch data for inferences on the world's marine fisheries. Fish Fish. 2012, 14, 293-311. [CrossRef]

19. Branch, T.A.; Jensen, O.P.; Ricard, D.; Ye, Y.M.; Hilborn, R. Contrasting global trends in marine fishery status obtained from catches and from stock assessments. Conserv. Biol. 2011, 25, 777-786. [CrossRef]

20. Dick, E.; MacCall, A.D. Depletion-Based Stock Reduction Analysis: A catch-based method for determining sustainable yields for data-poor fish stocks. Fish. Res. 2011, 110, 331-341. [CrossRef]

21. Martell, S.; Froese, R. A simple method for estimating MSY from catch and resilience. Fish Fish. 2013, 14, 504-514. [CrossRef]

22. MacCall, A.D. Depletion-corrected average catch: A simple formula for estimating sustainable yields in data-poor situations. ICES J. Mar. Sci. 2009, 66, 2267-2271. [CrossRef]

23. Froese, R.; Demirel, N.; Coro, G.; Kleisner, K.M.K.M.; Winker, H. Estimating fisheries reference points from catch and resilience. Fish Fish. 2016, 18, 506-526. [CrossRef]

24. Pella, J.J.; Tomlinson, P.K. A generalized stock production model. Inter-Am. Trop. Tuna Comm. Bull. 1969, $13,416-497$.

25. Winker, H.; Carvalho, F.; Kapur, M. JABBA: Just Another Bayesian Biomass Assessment. Fish. Res. 2018, 204, 275-288. [CrossRef]

26. Walters, C.J.; Martell, S.J.; Korman, J. A stochastic approach to stock reduction analysis. Can. J. Fish. Aquat. Sci. 2006, 63, 212-223. [CrossRef]

27. Thorson, J.T.; Rudd, M.B.; Winker, H. The case for estimating recruitment variation in data-moderate and data-poor age-structured models. Fish. Res. 2019, 217, 87-97. [CrossRef]

28. Methot, R.D.; Wetzel, C.R. Stock synthesis: A biological and statistical framework for fish stock assessment and fishery management. Fish. Res. 2013, 142, 86-99. [CrossRef]

29. Amoroso, R.O.; Pitcher, C.R.; Rijnsdorp, A.D.; McConnaughey, R.A.; Parma, A.M.; Suuronen, P.; Eigaard, O.R.; Bastardie, F.; Hintzen, N.T.; Althaus, F.; et al. Bottom trawl fishing footprints on the world's continental shelves. Proc. Natl. Acad. Sci. USA 2018, 115, E10275-E10282. [CrossRef]

30. FAO. Report of the Expert Consultation Workshop on the Development of Methodologies for the Global Assessment of Fish Stock Status, 4-6 February 2019; FAO Fisheries and Aquaculture Report No. 1262; License CC-BY-NC-SA 3.0 IGO; FAO: Rome, Italy, 2019.

31. Melnychuk, M.C.; Peterson, E.; Elliott, M.; Hilborn, R. Fisheries management impacts on target species status. Proc. Natl. Acad. Sci. USA 2017, 114, 178-183. [CrossRef]

32. Melnychuk, M.C.; Kurota, H.; Mace, P.M.; Pons, M.; Minto, C.; Osio, G.C.; Jensen, O.P.; de Moor, C.L.; Parma, A.M.; Little, L.R.; et al. Identifying management actions that promote sustainable fisheries. Nat. Sustain. 2021, 4, 440-449. [CrossRef]

33. Froese, R.; Winker, H.; Coro, G.; Demirel, N.; Tsikliras, A.C.; Dimarchopoulou, D.; Scarcella, G.; Palomares, M.L.D.; Dureuil, M.; Pauly, D. Estimating stock status from relative abundance and resilience. ICES J. Mar. Sci. 2019, 77, 527-538. [CrossRef] 